Skeletal muscle weakness, fatigue and sarcoidosis

\section{Skeletal muscle weakness, fatigue and sarcoidosis}

\section{U Costabel}

\section{It is important to remember that sarcoidosis is a complex multiorgan disease with multiple non-specific symptoms which go beyond the usual experience of chest physicians}

S arcoidosis is a multisystem granulomatous disorder that may involve many organs. Beside organ specific symptoms, non-specific constitutional complaints such as fatigue and general weakness are frequent. The granuloma formation in sarcoidosis is driven by an array of cytokines such as tumour necrosis factor (TNF)- $\alpha$, interleukin (IL)-6, interferon (INF)- $\gamma$ and others. High circulating levels of such cytokines have been found in sarcoidosis and may be responsible for provoking the constitutional symptoms fatigue and general weakness. $^{1-4}$

Fatigue and general weakness may be the reason why patients with sarcoidosis frequently experience exercise intolerance. $^{56}$ In one study $67 \%$ of patients with sarcoidosis terminated their peak exercise test due to "leg complaints". ${ }^{7}$ Skeletal muscle weakness may therefore be responsible for the limited exercise capacity, although quadriceps muscle force and handgrip force were found to be normal in the first study to investigate skeletal muscle force in patients with sarcoidosis, ${ }^{8}$ but most of these patients did not complain of fatigue.

In this issue of Thorax Spruit et a ${ }^{9}$ report that skeletal muscle weakness is present in patients with sarcoidosis who complain of fatigue, and that the presence of skeletal muscle weakness is associated with reduced health status as assessed by two questionnaires and also with reduced exercise capacity. In addition, quadriceps muscle force was inversely related to fatigue but not to the circulating levels of several proinflammatory cytokines. This would indicate that skeletal muscle weakness rather than the circulating cytokines are responsible for the complaints of fatigue in these patients. Interestingly, quadriceps muscle force was inversely related to the daily dose of corticosteroids in patients who received such treatment. This would imply that steroid induced myopathy may be one factor for reduced skeletal muscle function in sarcoidosis, although no differences in muscle strength were found in this study between patients who were taking steroids and those who were not.

Other factors may therefore be involved in the development of skeletal muscle weakness. One could be reduced physical activity which can induce general deconditioning in these patients who complain of fatigue, general malaise and depression, all of which will probably reduce the patients' physical activities. One major shortcoming of the paper by Spruit et al is that they did not investigate patients who did not complain of fatigue so it is difficult to generalise their findings to other patients with sarcoidosis. Would reduced skeletal muscle strength also be present in sarcoidosis patients without fatigue? We do not know since the number of patients studied in the first paper on skeletal muscle strength ${ }^{8}$ was probably too small to exclude a type II error.

Another factor contributing to the muscle weakness could be involvement of the skeletal muscle by sarcoidosis. ${ }^{10}$ The first published case of sarcoidosis of the skeletal muscles was reported as early as 1908 by Licharew who presented a girl with many palpable nodules in the muscle. ${ }^{11}$ In 1952 it was recognised that inactive sarcoidosis granulomas might frequently be found in clinically normal muscles. ${ }^{12}$ In 50 $75 \%$ of patients with sarcoidosis a muscle biopsy may detect non-caseating granulomas. ${ }^{12}{ }^{13}$ None of the patients in these screening studies had symptoms suggesting muscle involvement. This symptomless involvement resolves in the majority of cases during the natural course of the disease.

Clinically symptomatic skeletal muscle involvement in sarcoidosis is rare. There are two manifestations-acute polymyositis and chronic myopathy. Acute polymyositis has been reported in no more than 15 cases. ${ }^{10}$ The proximal shoulder and pelvic girdle muscles are most often involved and the condition is often associated with fever, erythema nodosum, myalgia, and polyarthralgia.
The levels of muscle enzymes such as creatine phosphokinase may be raised. Chronic myopathy is also rare. In a review of 800 patients with confirmed sarcoidosis Silverstein and Siltzbach ${ }^{14}$ found that only two had severe persistent sarcoid myopathy and, in the recent prospective ACCESS study, only one of 215 patients had initial muscle involvement. ${ }^{15}$ The symptoms are bilateral weakness and wasting of proximal muscles. Whereas corticosteroids are clearly indicated in these forms of clinically apparent muscle involvement, diagnostic difficulty may arise when myopathy occurs in patients with sarcoidosis under treatment with corticosteroids since corticosteroid myopathy has a similar distribution to that seen in sarcoid myopathy.

What are the practical issues emerging from the paper by Spruit et al? For differential diagnostic purposes, the muscle force tests are not useful since they do not discriminate between the possible different causes of skeletal muscle weakness in sarcoidosis. As previously mentioned, the causes may be limited physical activities, sarcoidosis involvement of the muscles, or corticosteroid myopathy. Ideally, we should have discriminating tests available which would be non-invasive and not depend on muscle biopsy. Less invasive techniques such as electromyography and nerval conductance velocity may differentiate between myopathy (either sarcoidosis or corticosteroid induced) and weakness due to limited exercise. Unfortunately, such neurological measurements for determining the cause of the skeletal muscle weakness were not included in the present study.

If we apply muscle force tests more frequently in patients with sarcoidosis and find reduced muscle strength, how should we manage such patients? We would expect that most of these patients also have the subjective complaint of fatigue, so the more general question would be how to manage skeletal muscle weakness and fatigue in sarcoidosis. Given the very rare clinically apparent skeletal muscle involvement by sarcoidosis granuloma and the very frequent subclinical muscle involvement which most probably represents harmless granuloma formation without impairment or wasting of the skeletal muscle, one would not start prednisone treatment for these patients but, rather, would recommend that they undergo controlled physical training programmes. As Spruit et al discuss, the positive effect of exercise training in chronic pulmonary and cardiac disease has been shown for other conditions and may reduce clinical symptoms of fatigue, anxiety, and depression. 
In this regard the great merit of the paper by Spruit et al is to remind the readers of Thorax that sarcoidosis is a complex multiorgan disease with multiple non-specific symptoms which go beyond the usual experience of chest physicians. The study underlines the importance of taking such patient complaints seriously, performing adequate investigations which may include muscle force tests, and giving them the appropriate treatment which would probably include an exercise programme to improve their physical activity which would also reduce the complaint of fatigue.

Thorax 2005;60:1-2.

doi: 10.1136/thx.2004.027342

Correspondence to: $\mathrm{Dr} U$ Costabel,

Ruhrlandklinik, Tueschener Weg 40, 45239

Essen, Germany; erj.costabel@t-online.de

\section{REFERENCES}

1 Asano $M$, Minagawa $T$, Ohimichi $M$, et al. Detection of endogenous cytokines in sera or in lymph nodes obtained from patients with sarcoidosis. Clin Exp Immunol 1991;84:92-6.

2 Shijubo N, Imai K, Shigehara K, et al. Soluble intercellular adhesion molecule-I (ICAM-1) in sera and bronchoalveolar lavage fluid of patients with idiopathic pulmonary fibrosis and pulmonary sarcoidosis. Clin Exp Immunol 1994;95: 156-61.

3 Prior C, Knight RA, Herold M, et al. Pulmonary sarcoidosis: patterns of cytokine release in vitro. Eur Respir J 1996;9:47-53.

4 Müller-Quernheim J. The cytokine network in sarcoidosis. Eur Cytokine Netw 1996;7:13-26.

5 Sharma OP. Fatigue and sarcoidosis. Eur Respir J 1999;13:713-4

6 Drent $M$, Wirnsberger RM, de Vries J, et al. Association of fatigue with an acute phase response in sarcoidosis. Eur Respir $J$ 1999;13:718-22.

7 Miller A, Brown LK, Sloane MF, et al. Cardiorespiratory responses to incremental exercise in sarcoidosis patients with normal spirometry. Chest 1995;107:323-9.
8 Wirnsberger $M$, Drent $M$, Hekelaar $N$, et al. Relationship between respiratory muscle function and quality of life in sarcoidosis. Eur Respir $J$ 1997; 10:1450-5.

9 Spruit MA, Thomeer MJ, Gosselink R, et al. Skeletal muscle weakness in patients with sarcoidosis and its relationship with exercise intolerance and reduced health status. Thorax 2005;60:32-8.

10 Rizzato G, Montemurro L. The locomotor system. In: James DG, ed. Sarcoidosis and other granulomatous disorders. New York: Marcel Dekker, 1994:349-73.

11 Licharew W. Moskauer venerologische und dermatologische Gesellschaft. Dermatol Zentralb/ 1908; 11:235.

12 Myers GB, Gottlieb AM, Mattman PE, et al. Joint and skeletal muscle manifestations in sarcoidosis. Am J Med 1952;12:161-9.

13 Wallace SL, Lattes R, Malia JP, et al. Muscle involvement in Boeck's sarcoid. Ann Intern Med 1958;48:497-51 1

14 Silverstein G, Siltzbach LE. Muscle involvement in sarcoidosis. Arch Neurol 1969;21:235-41.

15 Judson MA, Baughman RP, Thompson BW, et al. Two year prognosis of sarcoidosis: the ACCESS experience. Sarcoidosis Vasc Diffuse Lung Dis 2003;20:204-11.

see that the sum total exhalation of $\mathrm{NO}$ plus $\mathrm{NO}_{\mathrm{x}}$ is somewhat higher in $\mathrm{CF}$ than in controls, while the ratios of $\mathrm{NO}$ to $\mathrm{NO}_{\mathrm{x}}$ between the groups differ by a factor of 6 . If we consider the original source of all the nitrogen oxides in the lung to be from NOS (a somewhat risky assumption), then these data suggest that NO production is actually enhanced in the CF airway, not decreased as has at times been supposed. The fact that NO exhalation is low in CF then appears to result from overactive oxidative processes consuming NO.

A little more caution is needed here, as it is not clear how much of the gas phase $\mathrm{NO}$ available for exhaled measurements also contributes to the $\mathrm{EBC} \mathrm{NO}_{2}{ }^{-}$and $\mathrm{NO}_{3}{ }^{-}$levels. That work has yet to be done. But, as the authors note, it seems clear that $\mathrm{NO}_{\mathrm{x}}$ in $\mathrm{EBC}$ are not derived solely from $\mathrm{NO}$ gas dissolving and oxidising in the EBC ex vivo, as there are not enough available moles of NO gas in the exhaled air to provide for the levels of $\mathrm{NO}_{\mathrm{x}}$ seen in the EBC of CF patients.

Oxidation is one key sump for NO in the airway. Many believe oxidative processes to be a key mechanism by which "inflammation"-a generic term of uncertain meaning beyond the classical "rubor", "tumor", "calor" and "dolor"dutifully accomplishes its various missions. In general we consider NOS to be upregulated, acidity increased, and oxidation enhanced in inflammatory conditions. Inflammation might lead to higher or lower exhaled NO levels depending on the relative effects of the inflammation on NOS activity and acidic and oxidative stresses.

Ojoo et al have shown how these three chemical aspects of inflammation (NO, acid, and oxidants) can be monitored and distinguished. By measuring exhaled NO, exhaled acids, and exhaled
Increased exhalation of NO can occur from enhanced action of any of these with $\mathrm{CF}$, however, $\mathrm{NO}_{\mathrm{x}}$ are exhaled at the rate of $240 \mathrm{nmol} / \mathrm{h}$ compared with only $80 \mathrm{nmol} / \mathrm{h}$ exhaled as NO. One can 
$\mathrm{NO}_{\mathrm{x}}$ all together, one can make the following suppositions. If exhaled NO is high while exhaled $\mathrm{NO}_{\mathrm{x}}$ are low, then that particular airway inflammatory process has a relatively high ratio of airway NO production to oxidant activity. If exhaled NO is low while exhaled $\mathrm{NO}_{\mathrm{x}}$ are high, then the inflammation is relatively more dominated by oxidant stress than by NO production. When acidification is present, then NO will be both produced from $\mathrm{NO}_{2}{ }^{-}$and consumed by the higher oxidative activity that occurs in acidic conditions. ${ }^{3}$ Thus, EBC acidity stands by itself as an indicator of airway acid stress but not of NO production, and exhaled NO and $\mathrm{NO}_{\mathrm{x}}$ measured concurrently begin to elucidate the relative-not absoluteactivities of the NO production pathways and oxidative processes.

The same acidity that can convert aqueous $\mathrm{NO}_{2}{ }^{-}$into $\mathrm{NO}$ will also enhance oxidative activity in general, including the reaction of $\mathrm{NO}_{2}{ }^{-}$with the reductant, glutathione. This reaction forms S-nitrosoglutathione, an endogenous bronchodilator. ${ }^{5}$ To complicate matters, glutathione stores themselves can be depleted by excessive oxidative processes. Furthermore, the CF airway is commonly colonised with bacteria capable of reducing NO to ammonia, a process which can consume NO. ${ }^{6}$ Despite these complexities, efforts at measurement-and

\section{Concurrent trends in asthma and obesity \\ S Chinn}

\section{Is the concurrent rise in the prevalence of asthma and obesity a red herring?}

S tudies that have reported an association between asthma and obesity have mostly been single cross sectional studies, although an increased incidence of asthma in participants who were overweight at baseline has been reported in several longitudinal studies. The latter have contributed to the debate about the nature of the association, showing that the reverse causality explanation-that decreased physical activity as a result of asthma leads to increased weight-is not plausible. Cohort studies have the potential to determine whether increased symptoms of asthma are an immediate consequence of weight gain or, if not,

to establish the lag, but frequent follow up would be required. However, pure cohort studies cannot answer the question of how much of the increase in the prevalence of asthma in a population can be explained by an increase in obesity, as age and period are completely confounded. Repeated cross sectional studies in the same population are needed to answer this question.

\section{POPULATION SURVEYS}

Some countries have regular cross sectional population health surveys, such as the National Health and Nutrition Examination Surveys (NHANES) in the United States and the Health Survey for conscientious interpretation-of the chemistry of the airways helps us to recognise how acidic and oxidative processes, as well as NO chemistry, behave in various lung diseases. These processes are key mechanisms of inflammatory effect and are relatively easy therapeutic targets.

Another point is worth noting. were not significantly lower on average during acute respiratory exacerbations. This statement reminds us that "no statistically significant difference" is not synonymous with "no difference". Given that EBC $\mathrm{pH}$ was significantly lower in the exacerbating group relative to the stable group, the lay reader might conclude that EBC $\mathrm{pH}$ is more useful than spirometry at identifying CF exacerbations. A more cautious and probably wiser interpretation might be that-together-spirometry, EBC pH, exhaled $\mathrm{NO}$, and $\mathrm{NO}_{2}{ }^{-}$and $\mathrm{NO}_{3}{ }^{-}$assays provide a more comprehensive and true picture of airway disease than any single measurement. EBC pH informs us about airway acid stress. $\mathrm{NO}$ and $\mathrm{NO}_{\mathrm{x}}$, when measured together, inform us about NO production and oxidative burden. Spirometry provides physiological data. Of course, none of these tests delineates perfectly the amount of "inflammation". But then, in the absence of a unifying definition of inflammation, no one test possibly could. Spirometric values in CF in this study

Thorax 2005;60:2-3.

doi: $10.1136 /$ thx.2004.024364

Correspondence to: $\mathrm{Dr} J$ Hunt, Division of Pediatric Respiratory Medicine, Box 800386, University of Virginia, Charlottesville, VA 22908, USA; ifh2m@virginia.edu

Competing interest statement: (1) The University of Virginia owns intellectual property relating to measurement of exhaled breath condensate $\mathrm{pH}$. (2) The author is a founder of Respiratory Research Inc, a company that builds exhaled breath condensate collection equipment. (3) The author is an inventor on US and international patents relating to measurement of exhaled breath condensate nitrogen oxides, but has no financial interest in these patents.

\section{REFERENCES}

1 Ojoo JC, Mulrennan SA, Kastelik JA, et al. Exhaled breath condensate $\mathrm{pH}$ and exhaled nitric oxide in allergic asthma and in cystic fibrosis. Thorax 2005;60:22-6.

2 Lee KH, Rico P, Billiar TR, et al. Nitric oxide production after acute, unilateral hydrochloric acid-induced lung injury in a canine model. Crit Care Med 1998;26:2042-7.

3 Weitzberg E, Lundberg JO. Nonenzymatic nitric oxide production in humans. Nitric Oxide 1998;2:1-7

4 Hunt JF, Fang K, Malik R, et al. Endogenous airway acidification. Implications for asthma pathophysiology. Am J Respir Crit Care Med 2000;161:694-9.

5 Gaston B, Drazen JM, Loscalzo J, et al. The biology of nitrogen oxides in the airways. Am J Respir Crit Care Med 1994;149:538-51.

6 Gaston B, Ratjen F, Vaughan JW, et al. Nitrogen redox balance in the cystic fibrosis airway: effects of antipseudomonal therapy. Am J Respir Crit Care Med 2002; 165:387-90.

England. Such surveys routinely include height and weight, as increased body mass index (BMI) in adults is a risk factor for a number of diseases, but the Health Survey for England has included respiratory symptoms only in selected surveys. Increases in asthma and in overweight (BMI $25-<30 \mathrm{~kg} / \mathrm{m}^{2}$ ) or obesity (BMI $\geqslant 30 \mathrm{~kg} / \mathrm{m}^{2}$ ) have been reported many times over the last 20 years, but rarely in the same study. This has led to speculation that the rise in obesity might explain some of the increase in the prevalence of asthma, but there has been little direct evidence to support or refute the hypothesis. ${ }^{1-4}$

\section{REPEATED CROSS SECTIONAL STUDIES IN CHILDREN}

Some direct evidence is provided in this issue by Wickens et al. ${ }^{5}$ They studied comparable groups of 11-12 year old children in New Zealand in 1989 and 2000 and found an increased prevalence of symptoms, asthma, and medication in 2000 compared with 1989, but little difference in the corresponding odds ratios unadjusted or adjusted for BMI. They also found a stronger relation between most outcomes and BMI in 2000 than in 1989, particularly for wheeze and medication in the last 12 months, 
which remained statistically significant in the 2000 data after adjustment for a number of potential confounders.

The results are similar to those of Chinn and Rona from the National Study of Health and Growth (NSHG), who found that virtually none of the increase in wheeze or asthma in 8 and 9 year old children in the UK from 1982 to 1994 was explained by the rise in overweight and obesity, and also reported a stronger association between asthma and obesity in the later than in the earlier year in children aged 5-11 years. The UK and New Zealand studies were each carried out in a school population with symptoms and medication reported by parent or caregiver. Height and weight were measured, unlike in many adult studies from which an association between asthma and obesity has been reported. However, in children BMI is a poorer measure of fatness than skinfold thickness. In the NSHG there was a weaker association of asthma and persistent wheeze with skinfold measurements than with BMI. ${ }^{7}$

\section{IS THE ASSOCIATION RECENT IN CHILDREN?}

Wickens et al considered that there was insufficient evidence to conclude that the association in children is of recent origin. ${ }^{5}$ They argued that comparisons of studies over time are hampered by changing definitions of asthma. Certainly, comparison is difficult as the lack of definition of overweight and obesity in children before 2000 led to a variation in reporting results. However, the NSHG had identical symptom questions over time, and there is no doubt that the association of persistent wheeze with BMI was stronger in the representative sample in 1993/1994 than in $1977 . .^{78}$ Neither the $\mathrm{NSHG}^{6}$ nor the New Zealand study ${ }^{5}$ was able to show an interaction between year of survey and the association of asthma and BMI, possibly due to lack of power but, taken together, the evidence is strengthened that the association in children is recent.

\section{EVIDENCE IN ADULTS}

No direct information on the BMI adjusted trend in the prevalence of asthma has been published in adults, although data on asthma and BMI were obtained in NHANES (at least in surveys II and III). ${ }^{910}$ There is also a lack of information on when the association between asthma and obesity arose in adults, or whether it is long standing but only noticed once obesity became more prevalent. The earliest studies to report an association were carried out in the early 1980s and were studies of chronic disease in general. ${ }^{11}{ }^{12}$
A rough estimate of the maximum likely effect on the rise in asthma can be obtained, for a given increase in prevalence of overweight and obesity, on the assumption that the association is causal. For example, a change in BMI distribution from $85 \%$ normal weight, $10 \%$ overweight and $5 \%$ obese to $75 \%$ normal, 15\% overweight and 10\% obese would, at most, increase the prevalence of asthma by $6.8 \%$ over baseline, given the published estimates of relative risks. A baseline prevalence of $5 \%$ would increase to $5.3 \%$, a very modest rise compared with most of those reported. So we should not expect the rise in overweight and obesity to explain more than a very small amount of the increase in asthma.

\section{EVIDENCE FROM TRENDS}

Ideally, trends should be calculated from more than two time points. Long term trends in asthma and BMI can show whether the increase in asthma started close to, or soon after, the beginning of the major increase in obesity. Parallel trends, or points of inflexion close in time, tell us little in themselves other than that a direct causal role is possible. The contrary-especially a reversal of one trend in the absence of a change in the other-is evidence against a direct causal relation provided the data are representative of the same populations. There is now some evidence for a recent slowing down or cessation of the increase in asthma in children and in adults. ${ }^{13-16}$ Data on BMI were not collected in the individual studies and three of the studies were carried out in selected areas. ${ }^{13} 1416$ The fourth study used data from 1990 to 1998 from the UK General Practice Research Database and showed a levelling off of trends in managed asthma from about $1995 .{ }^{15}$ Recent trends in BMI in Italy or Switzerland are not available but in England there is evidence from the Health Survey for England for an increase in obesity in adults from 1993 to 1998. While England is not the whole of the UK, and neither study may be fully representative as the General Practice Research Database may have practice participation bias and the Health Survey for England individual non-response bias, taken together the results suggest that the trends are not parallel.

\section{CONCURRENT TRENDS - A RED HERRING?}

There is little doubt that there was a concurrent rise in the prevalence of asthma and obesity in many developed countries in the 1980s and early 1990s, and this led to the speculation that the increase in obesity might explain some of the trend in asthma. Even without more direct evidence that the trend in overweight and obesity explains little or none of the trend in asthma, we can conclude that controlling the weight of the population would do little to reduce the prevalence of asthma as the associated relative risks are too small. However, it may take more direct evidence to convince the whole of the scientific community of this and, if further data exist to establish whether the association is recent or long standing in adults, this would be of interest. A long standing association in adults would contrast with that in children and would make changes in the population in diet and physical activity less likely as an explanation.

Thorax 2005;60:3-4.

doi: 10.1136/thx.2004.031161

Correspondence to: Professor S Chinn, Department of Public Health Sciences, King's College London, London SEI 3QD, UK; sue.chinn@kcl.ac.uk

\section{REFERENCES}

1 Shaheen SO. Obesity and asthma: cause for concern? Clin Exp Allergy 1999;29:291-3.

2 Camargo CA, Weiss ST, Zhang S, et al. Prospective study of body mass index, weight change, and risk of adult-onset asthma in women. Arch Intern Med 1999;159:2513-4.

3 Castro-Rodriguez JA, Holberg CJ, Morgan WJ, et al. Increased incidence of asthmalike symptoms in girls who become overweight or obese during the school years. Am J Respir Crit Care Med 2001;163:1344-9.

4 Gilliland FD, Berhane K, Islam T, et al. Obesity and the risk of newly diagnosed asthma in schoolage children. Am J Epidemiol 2003;158:406-15.

5 Wickens K, Barry D, Friezema A, et al. Obesity and asthma in 11-12 year old New Zealand children in 1989 and 2000. Thorax 2005;60:7-12.

6 Chinn S, Rona RJ. Can the increase in body mass index explain the rising trend in asthma in children? Thorax 2001;56:845-50.

7 Figueroa-Muñoz Jl, Chinn S, Rona RJ. Association between obesity and asthma in 4 to 11 year old children in the UK. Thorax 2001;56:133-7.

8 Somerville SM, Rona RJ, Chinn S. Obesity and respiratory symptoms in primary school. Arch Dis Child 1984;59:940-4.

9 Flegal KM, Carroll MD, Kuczmarski RJ, et al. Overweight and obesity in the United States: prevalence and trends, 1960-1994. Int J Obesity 1998:22:39-47.

$10 \mathrm{Kwon} \mathrm{HL}$, Belanger K, Bracken MB. Asthma prevalence among pregnant and childbearing women in the United States: estimates from national health surveys. Ann Epidemiol 2003;13:317-24.

11 Seidell JC, de Groot LCPGM, van Sonsbeek JLA, et al. Associations of moderate and severe overweight with self-reported illness and medical care in Dutch adults. Am J Public Health 1986:76:264-9.

12 Negri E, Pagano R, Decarli A, et al. Body weight and the prevalence of chronic disease. J Epidemiol Community Health 1988;42:24-9.

13 Ronchetti $R$, Villla $M$, Rota $R$, et al. Is the increase in childhood asthma coming to an end? Findings from three surveys of schoolchildren in Rome, Italy. Eur Respir J 2001;17:881-6.

14 Verlato G, Corsico A, Villani S, et al. Is the prevalence of adult asthma and allergic rhinitis still increasing? Results of an Italian study. J Allergy Clin Immunol 2003;1 11:1232-8.

15 Soriano JB, Kiri VA, Maier WC, et al. Increasing prevalence of asthma in UK primary care during the 1990s. Int J Tuberc Lung Dis 2003:7:415-21.

16 Braun-Fahrländer C, Gassner M, Gize L, et al. No further increase in asthma, hay fever and atopic sensitisation in adolescents living in Switzerland. Eur Respir J 2004;23:407-13. 
Clinical remission of asthma

\section{Clinical remission of asthma: what lies beyond? \\ S Guerra}

\section{Further evidence suggests that "complete" remission of childhood asthma may be the exception rather than the rule}

A sthma can be a debilitating disease with a major impact on quality of life and an increased risk of developing severe airway remodelling and non-fully reversible airflow obstruction. In the last decades, effective pharmacological treatments and management strategies have been developed for this disease. Yet, at the present time, asthma remains a treatable but not a curable disease.

It is not therefore surprising that much effort is being put into studying the risk factors associated with the inception and progression of asthma, since understanding these factors represents the first necessary step for developing effective prevention strategies. In this respect, the increase in the incidence and prevalence of asthma over the last decades has not only produced a general awareness of the public health burden associated with the disease, but it has also provided the scientific rationale for expecting a major role of environmental factors in its development. However, the epidemiological identification of these disease determinants has not proved to be an easy task. While profiles of risk factors for asthma have been identified, the exact mechanisms by which they operate are far from being fully understood for many (or most) of them. In addition, many of these factors are likely to be involved in complex gene-by-environment interactions and to be differentially linked to different asthma phenotypes. This complexity has limited the implementation of effective interventions to reduce the incidence of asthma and, to date, there is no conclusive programme of primary prevention for this disease.

To complicate things even more, remarkable paradoxes have emerged from recent research. Factors that are known to exacerbate or trigger asthma, such as exposure to endotoxin ${ }^{1}$ or pets, ${ }^{2}$ appear to be somewhat protective if they occur early in life. Similarly, while allergen avoidance is undoubtedly a beneficial and essential part of management strategies in atopic asthma, it does not appear to be as effective in protecting against onset of new asthma. Taken together, these observations strongly suggest that factors that determine the inception of asthma may differ from those that affect its progression, and reinforce the unique importance of studies that follow subjects with asthma over time. Observational and intervention studies on selected cohorts of asthmatics have indeed provided critical contributions to our understanding of the factors influencing the clinical course of the disease, and this evidence has affected to a large extent the way we currently treat and manage asthma.

Studies on asthmatic cohorts can also help to elucidate the natural history and long term outcomes of the disease. From a clinical standpoint, this is not an issue of secondary importance. Asthma is a variable disease that can persist, remit conclusively, or present any possible combination of remissions and relapses over time. Clearly, the long term sequelae of the disease on lung health are likely to be quite different across these different patterns. In this framework, longitudinal studies assessing the outcome of childhood asthma into adult life hold particular interest for two main reasons. Firstly, in the vast majority of cases asthma has its onset in childhood. Therefore, even though adult onset asthma is frequently associated with severe and difficult to treat forms of the disease, asthma initiated in childhood continues to have a greater public health impact at the population level. Secondly, asthma may lead in the long term to severe airway remodelling and, possibly, to the development of chronic airflow obstruction. These observations raise critical questions on whether this course is more likely to occur in the persistent forms of asthma, whether subjects predisposed to this progression can be identified at early stages of their disease, and whether optimal asthma management can be beneficial against disease progression. Longitudinal studies following cohorts of participants over a wide age span between childhood and adulthood represent our best tool to answer these questions.
In a recent issue of Thorax Vonk et al reported evidence from such a cohort of 119 allergic asthmatic children (age 5-14 years) enrolled in an outpatient clinic in the late 1960s and re-evaluated in a follow up visit approximately 30 years later (age 32-42 years). Although the relatively small sample size suggests caution in interpreting these data, this study provides cogent findings that are consistent with those from previous longitudinal studies and may have important implications for the search of optimal management strategies in asthma.

Firstly, childhood asthma appeared to remit clinically (no reported active symptoms and no use of inhaled corticosteroids) in adult age "only" in approximately $50 \%$ of the cases. In interpreting these results, the selective nature of this cohort of outpatients should be kept in mind. It is known that children with asthma selected in the clinical setting are likely to differ from population based samples of asthmatics in many respects, particularly in terms of increased severity of the disease-a known risk factor for persistence of symptoms into adulthood. Rates of asthma persistence are therefore expected to be higher in clinic based than in population based cohorts.

Consistently, findings from several long term longitudinal cohorts ${ }^{4-7}$ indicate that, in the general population, among subjects who had episodes of wheezing in childhood, approximately one third report active asthma symptoms when surveyed in their young to mid adult life. However, this proportion is remarkably higher, even in population based studies, among subjects whose childhood asthma was severe or characterised by frequent wheezing episodes. This concept is best illustrated by data from the Melbourne Asthma Study ${ }^{8}$ in which several groups of school age children were enrolled based on their wheezing/asthma history and followed over time into their adulthood. At age $42,15 \%$ of controls reported wheezing episodes in the last 3 years compared with $40 \%$ of the subjects who, during childhood, had wheezing associated with bronchitis or respiratory tract infection. However, wheezing episodes were reported by up to $70 \%$ and $90 \%$ of subjects who during childhood had fulfilled the criteria for asthma and severe asthma, respectively.

All evidence considered, it appears plausible to conclude that most children with moderate to severe forms of asthma will experience clinically active disease at least in some periods of their adult life. This important conclusion becomes even more striking when we consider a second finding from the 
study by Vonk et $a l^{3}$ : most cases of asthma in clinical remission (in that study, up to $57 \%$ ) still show bronchial hyperresponsiveness and/or reduced lung function. This finding supports a clear distinction between clinical remission of asthma (that is, absence of symptoms and medication use) and complete remission of asthma. The implications of this distinction are quite relevant, given that the simple absence of symptoms is the most commonly used criterion for defining asthma remission in epidemiology and it is also used to a large extent in clinical algorithms for managing the disease.

Does the presence of bronchial hyperresponsiveness and reduced lung function among these apparently remitting asthma cases represent structural sequelae of the disease? Or does it represent an underlying inflammatory process still active in the airways? There are arguments to support both these scenarios. Most likely, in many cases of clinical remission of asthma an ongoing airway inflammation is present which can interact with-and possibly enhanceairway remodelling and structural changes of the lungs. Recent studies ${ }^{9-11}$ have shown that, when compared with healthy controls, children and adolescents who have apparently outgrown their asthma related symptoms do show increased markers of airway inflammation. Eosinophils and interleukin (IL)-5 in bronchial biopsy tissue, the percentage of eosinophils in bronchoalveolar lavage fluid, exhaled nitric oxide levels, and bronchial responsiveness to adenosine-5'-monophosphate all appear consistently higher in subjects with remitting asthma than in controls. ${ }^{9-11}$ This evidence raises the question whether a long term anti-inflammatory treatment should be considered in these cases of subclinical asthma. At the present time we do not have a conclusive answer to this question. However, the accumulating evidence on inflammation and airway remodelling in remitting asthma strongly argues for at least monitoring patients with asthma in clinical remission over time, possibly with periodic assessments of lung function, bronchial hyperresponsiveness, and other markers of inflammation.

Another interesting finding from the study by Vonk and colleagues ${ }^{3}$ is that reduced lung function in childhood was a significant risk factor for the persistence of asthma and airflow limitation into adulthood, an association described quite consistently across previous large longitudinal studies. ${ }^{56}$ Early deficits in lung function among children with asthma may be related to several factors, including early airway remodelling or impaired lung growth. Regardless of the nature of these factors, the fact that these deficits track over time implies that children with persistent asthma will be most often destined to have lower lung function than their peers in adult life. In addition, among subjects with long term asthma, these deficits in lung function may eventually lead to a non-fully reversible airflow obstruction-a key functional feature for the definition of chronic obstructive pulmonary disease (COPD). ${ }^{12}$ The co-existence of asthma and COPD diagnoses in the same subject appears quite frequently in adults $^{13}$ and appears to be associated with increased mortality. ${ }^{14}$ It is debatable whether subjects with asthma are at increased risk for COPD as a result of the clinical progression of the disease (that is, long term sequelae of the airway inflammation and remodelling associated with asthma) or as a result of an increased susceptibility to lung damage by noxious agents. ${ }^{15}$ This is a vital question in terms of optimal prevention, treatment, and management of patients with co-existing signs of asthma and COPD. The scenario of a clinical progression of asthma into COPD is supported by a large body of evidence, including the reported correlation among asthmatics between the degree of airway remodelling associated with the disease and the presence of COPD-like functional signs such as irreversible airflow obstruction. ${ }^{16}{ }^{17}$ However, it is tempting to speculate that the tracking of reduced lung function over time shown by subjects who had severe childhood asthma may also play an important role in increasing the susceptibility of these subjects in adult life to noxious agents including cigarette smoking, and in lowering their threshold for a clinically relevant lung damage in response to these agents. At the present time this remains a largely unsolved question.

In this framework, the results on asthma persistence provided by Vonk and colleagues ${ }^{3}$ reinforce the clinical relevance of understanding the long term sequelae of this disease. New evidence from longitudinal cohorts of asthmatic children entering their mid adult life will contribute to determining the structural and functional consequences of persistent asthma on the lungs and dissecting further the long hypothesised link between childhood asthma and COPD in adulthood. ${ }^{18} 19$

Thorax 2005;60:5-6.

doi: $10.1136 /$ thx. 2004.033480
Correspondence to: $\operatorname{Dr} S$ Guerra, Arizona Respiratory Center, University of Arizona, 1501 N. Campbell Ave, P O Box 245030, Tucson, AZ 85724-5030, USA; sguerra@ arc.arizona.edu

Dr Guerra is supported in part by a Grant Award from the American Thoracic Society/ Alpha One Foundation.

\section{REFERENCES}

1 Liu AH. Endotoxin exposure in allergy and asthma: reconciling a paradox. J Allergy Clin Immunol 2002; 109:379-92.

2 Simpson A, Custovic A. Early pet exposure: friend or foe? Curr Opin Allergy Clin Immunol 2003;3:7-14.

3 Vonk JM, Postma DS, Boezen HM, et al. Childhood factors associated with asthma remission after 30 year follow up. Thorax 2004:59:925-9.

4 Horak E, Lanigan A, Roberts $M$, et al. Longitudinal study of childhood wheezy bronchitis and asthma: outcome at age 42. BMJ 2003;326:422-3.

5 Sears MR, Greene JM, Willan AR, et al. A longitudinal, population-based, cohort study of childhood asthma followed to adulthood. N Engl J Med 2003;349:1414-22.

6 Jenkins MA, Hopper JL, Bowes G, et al. Factors in childhood as predictors of asthma in adult life. BMJ 1994;309:90-3.

7 Strachan DP, Butland BK, Anderson HR. Incidence and prognosis of asthma and wheezing illness from early childhood to age 33 in a national British cohort. BMJ 1996;312:1195-9.

8 Phelan PD, Robertson CF, Olinsky A. The Melbourne Asthma Study: 1964-1999. J Allergy Clin Immunol 2002; 109:189-94.

9 van Den Toorn LM, Prins JB, Overbeek SE, et al. Adolescents in clinical remission of atopic asthma have elevated exhaled nitric oxide levels and bronchial hyperresponsiveness. Am J Respir Crit Care Med 2000; 162:953-7.

10 van den Toorn LM, Overbeek SE, de Jongste JC, et al. Airway inflammation is present during clinical remission of atopic asthma. Am J Respir Crit Care Med 2001;164:2107-13.

11 Warke TJ, Fitch PS, Brown V, et al. Outgrown asthma does not mean no airways inflammation. Eur Respir J 2002; 19:284-7.

12 Pauwels RA, Buist AS, Calverley PM, et al. Global strategy for the diagnosis, management, and prevention of chronic obstructive pulmonary disease. NHLBI/WHO Global Initiative for Chronic Obstructive Lung Disease (GOLD) Workshop summary. Am J Respir Crit Care Med 2001;163:1256-76.

13 Soriano JB, Davis KJ, Coleman B, et al. The proportional Venn diagram of obstructive lung disease: two approximations from the United States and the United Kingdom. Chest 2003; 124:474-81.

14 Meyer PA, Mannino DM, Redd SC, et al. Characteristics of adults dying with COPD. Chest 2002; 122:2003-8.

15 Silva GE, Sherrill DL, Guerra S, et al. Asthma as a risk factor for COPD in a longitudinal study. Chest 2004; 126:59-65.

16 Benayoun L, Druilhe A, Dombret MC, et al. Airway structural alterations selectively associated with severe asthma. Am J Respir Crit Care Med 2003; 167:1360-8.

17 Gono H, Fujimoto K, Kawakami S, et al. Evaluation of airway wall thickness and air trapping by HRCT in asymptomatic asthma. Eur Respir J 2003:22:965-71.

18 Burrows B, Knudson RJ, Lebowitz MD. The relationship of childhood respiratory illness to adult obstructive airway disease. Am Rev Respir Dis 1977;115:751-60.

19 Orie NGM, Sluiter HJ, de Vries K, et al. The host factor in bronchits. In: Orie NGM, Sluiter HJ, eds. Bronchitis: an international symposium. Assen, The Netherlands: Royal vanGorcum, 1961. 